\title{
The application of Langmuir-Blodgett technique in preparation of the macroporous titania coatings
}

\author{
Ireneusz Piwoński • Aneta Kisielewska • \\ Jacek Marczak
}

Published online: 21 June 2013

(c) The Author(s) 2013. This article is published with open access at Springerlink.com

\begin{abstract}
The aim of this work was preparation of the macroporous titania coatings with the use of the sol-gel process and poly(methylmetacrylate) beads as a template. The effectiveness of the Langmuir-Blodgett (LB) and dipcoating (DC) methods in deposition of polymer beads on the silicon wafers was compared. Resulted polymer layers and final porous titania coatings were analyzed with the use of the atomic force microscope. It was found, that application of the LB is possible only when arachidic acid is present in the subphase. It should be highlighted, that the application of the LB method is the novelty between the methods of the polymer beads arrangement having the diameter of 200-300 nm. Main factors which influence the structure and the arrangement of polymer templates were the concentration of the polymer suspension and the rate of the substrate immersion/withdrawal from the suspension. We established, that the optimal concentrations for preparation of polymer templates, exhibiting good arrangement of individual beads, were 0.5 and $6 \%$ for LB and DC methods, respectively. The size of pores of the obtained macroporous titania (200-330 nm) corresponds well with the size of the polymer beads used as the template $(200-235 \mathrm{~nm})$.
\end{abstract}

Keywords $\mathrm{TiO}_{2} \cdot$ Macroporous coatings $\cdot$ Sol-gel chemistry · Atomic force microscopy

I. Piwoński $(\varangle) \cdot$ A. Kisielewska · J. Marczak

Department of Materials Technology and Chemistry, University of Łódź, Pomorska 163, 90-236 Łódź, Poland

e-mail: irek@uni.lodz.pl

\section{Introduction}

Ceramic macroporous materials find wide application in catalysis, separation techniques, photonic crystals and optoelectronics. These structures exhibit highly homogenous distribution of spherical pores having the diameter over $50 \mathrm{~nm}$. In the preparation of macroporous solids, polymer colloid spheres, serving as the design template are used. The diameter of pores can be controlled by precisely defined size of the template (polymer spheres). One of the members of the macroporous solids family are three dimensional ordered materials (3DOM). These materials exhibit high surface area and low dielectric constant. They offer unique optical properties like controlled light propagation in the UV and visible light wavelength range. These properties can be combined with physicochemical features of materials having large potential in nanotechnological applications-e.g. titanium dioxide. Mainly due to its photoactivity and high refractive index $(>2.5)$.

Titanium dioxide can be also prepared as macroporous structures providing building blocks to the periodic ordered structures, having different refractive index areas and photonic band gap (photonic crystals). In photonic crystals, photons having energy in the range of the photonic band gap cannot propagate, independent on the direction of propagation.

Qian et al. reported the preparation of polymer latex spheres organized in the form of monolayer at the air/water interface deposited onto glass slides through the Langmuir-Blodgett (LB) technique [1]. The monolayer of poly(styrene) (PS) beads prepared in such a way were deposited on glass, optical fibers and decorative materials exhibiting large scale defect-free areas.

In similar study amphiphilic silica spheres prepared by hydrolysis of tetraethyl orthosilicate (TEOS) and modified 
by grafting vinyl or amino groups were organized as a stable Langmuir film at the water-air interface [2]. Langmuir-Blodgett method was applied to produce a colloidal crystal materials consisted of a defined number of layers of silica spheres having different sizes. Potential applications of these types of 3D structures include low-threshold lasers or templates for macroporous materials.

There are many examples of the preparation of macroporous materials using silica or polymer spheres as templates. Three-dimensionally ordered macroporous titania obtained by sol-gel reactions and imprinted on latex particles was in the centre of interest of Torralvo et al. [3]. PS or polystyrene co-polymerized with other compounds beads were used as templates resulting in preparation of macro-mesoporous titania structures. Authors were focused on optimization of the surface area, pore size and mesopore distribution inside the macropore walls by selecting appropriate sol-gel conditions, type of the template and infiltration process.

In another study macroporous titania and zirconia were obtained by the template-assisted colloidal processing technique or hetero-coagulation of template/ceramic particle colloidal processing [4]. Two methods were proposed. In the first method, polymer spheres were prepared as a template, followed by the infiltration with alkoxide precursors and the removal of the template by calcination at the appropriate temperatures. In the second method coreshell polymer/ceramic composites were obtained by mixing the oppositely charged two suspensions via electrostatic attraction following by filtration and calcination. Both methods yielded in well-ordered macroporous ceramics. In similar way ceramics consisting of $\mathrm{SiO}_{2}, \mathrm{TiO}_{2}$ and $\mathrm{ZrO}_{2}$ have been fabricated also via a template-assisted colloidal processing technique [5].

Preparation and catalytic properties of macroporous sulfated zirconia was reported by Stein et al. [6]. In this work latex spheres were infiltrated with the precursor solutions containing varying $\mathrm{SO}_{4} / \mathrm{Zr}$ molar ratios. More accurate control of the material properties was achieved by application of sulfates, which retarded the crystallization and crystal growth as well as the appropriate calcination temperatures. These properties included macropore shrinkage, grain size, specific surface area, composition and periodic order of the material.

Macroporous alumina was obtained by mixing alumina sol with PS particles as templates by Dong et al. [7]. This study showed that the Zeta potential plays important role in the tuning of the final structure of the macroporous materials. The Zeta potential of PS beads can be easily switched by its modification, resulting in obtaining vesicles free materials having better pores interconnectivity.

The review of synthesis of highly ordered macroporous materials composed of oxides of $\mathrm{Si}, \mathrm{Ti}, \mathrm{Zr}, \mathrm{Al}, \mathrm{W}, \mathrm{Fe}$ and
Sb templated around PS (latex) spheres were presented by Holland et al. [8, 9]. Detailed characterization of obtained solids demonstrated that macroporous materials can by synthesized in various compositions, depending on applied precursor. As well, it was shown that such parameters like void packing arrangements, wall thickness and porosity can by controlled in order to adjust the properties of these materials to such applications like photonic crystals, catalysis, large molecule separation processes etc.

In similar study bioactive sol-gel glasses with or without 3DOM structures were prepared [10]. The influence of the porous structure on "in vitro" activities toward hydroxycarbonate apatite mineralization from simulated body fluid was studied. It was found, that the 3DOM bioactive glass exhibited faster growth of apatite than the control sample without porosity due to more accessible porous network.

Between several applications of 3DOM materials, its use as membranes seems to be the natural choice. Fabrication of porous membranes having three-dimensional network was reported by Xia et al. [11]. Porous membranes were obtained by injection of an aqueous dispersion of monodispersed PS (or silica) beads into a specially designed cell and assembled into an opaline array under external gas pressure and sonication. After drying, the void spaces among the spheres were filled with a liquid sol-gel solution. Described procedure can be also extended to fabrication of variety of materials, including a UV-curable poly(acrylate-methacrylate) copolymer (PAMC), UV-curable polyurethanes or oxide ceramics such as $\mathrm{SiO}_{2}$ or $\mathrm{TiO}_{2}$.

Finally, macroporous metals can be also prepared. Yan et al. [12] reported a general procedure for synthesizing the macroporous metals with 250-500 nm voids surrounded by nanocrystalline metal walls. The method is based on the precipitation and chemical conversion of the precursors to a macroporous metal oxide in the presence of the template. The metal oxide can be further converted to a purely metallic, ordered macroporous solid by reduction in hydrogen.

The aim of our work was the investigation of parameters, which are crucial in controllable preparation of macroporous titania coatings with the use of the polymer beads as a template.

We demonstrate the application of Langmuir-Blodgett technique in controlled deposition of poly(methylmetacrylate) (PMMA) beads on the silicon wafers. For comparative studies (as alternative method), dip-coating deposition of PMMA beads from their suspension solutions was applied. PMMA colloid spheres were synthesized by emulsifier-free polymerization. Polymer beads deposited in such a manner were used as a template for the preparation of the macroporous titania coatings. As far as we know, the application of Langmuir-Blodgett technique in preparation 
of colloid crystals is very rare. Fabrication of the PMMA molecules onto the solid substrates in order to form Langmuir-Blodgett (LB) thin films was studied by Çapan [13]. In our work we go one step further, showing the application of the LB films consisted of PMMA beads, in the preparation of macrporous titania coatings.

\section{Experimental}

\subsection{Materials}

The following reagents and materials were used in our experiments:

- Methyl methacrylate (MMA, 99 \%), Sigma-Aldrich.

- 2,2'-azobis(2-methylpropionamidine) dihydrochloride (97\%), Sigma-Aldrich.

- Titanium (IV) isopropoxide (97\%), ABCR GmbH\&Co. Karlsruhe, Germany.

- Isopropyl alcohol (99.5\%), POCh S.A., Gliwice, Poland.

- Arachidic acid (97\%), Sigma-Aldrich.

- Deionized water.

- Silicon wafers-Si (100)_Cemat Silicon, Warsaw, Poland.

All reagents were used without further purification.

\subsection{Synthesis of PMMA spheres}

Poly(methylmethacrylate) (PMMA) colloid spheres having various sizes were synthesized by emulsifier-free polymerization in two variants described below at various conditions. The synthesis is based on the method described by Zou [14].

\subsubsection{Synthesis I}

The synthesis was carried out in a 4-necked round-bottomed flask ( $0.5 \mathrm{~L}$ volume), provided with mechanical stirrer, thermometer, water-cooled reflux condenser, nitrogen bubbler and additional funnel. The nitrogen was slowly bubbled into the flask equipped with mechanical stirring (700 rpm), filled with distilled water $(0.250 \mathrm{~L})$ and heated to $85{ }^{\circ} \mathrm{C}$. Next, methyl methacrylate (MMA, 0.012 L) was slowly added using additional funnel. When the appropriate temperature was achieved, an azo initiator: 2,2'-azobis(2-methylpropionamidine) dihydrochloride (0.075 g) was added, in order to initiate polymerization of the MMA. The temperature of the mixture was kept constant $\left(85^{\circ} \mathrm{C}\right)$ for $2 \mathrm{~h}$ under stirring. Larger agglomerates were filtered off and removed from the water suspension of the resulted polymer spheres using cotton wool. Subsequently, filtered suspension was centrifuged $(3,000 \mathrm{rpm})$ for $15 \mathrm{~min}$ and decanted-fraction 1. Obtained precipitate of polymer spheres were dried in air at room temperature. The rest of the suspension was centrifuged once again $(6,000 \mathrm{rpm})$ for 10 min-fraction 2 . In this way two white precipitates fractions of polymer spheres having different sizes were obtained.

\subsubsection{Synthesis II}

The Synthesis II was the modification of the synthesis I. It was performed in order to obtain PMMA spheres having larger size than spheres obtained in the synthesis I. The main difference was the smaller amount of initiator: 2,2'azobis(2-methylpropionamidine) dihydrochloride $(0.060 \mathrm{~g})$, and lower intensity of stirring-350 rpm.

\subsection{Synthesis of PMMA templates}

Dry PMMA spheres were dispersed in deionized water to get an aqueous suspensions having defined concentrations. Suspensions were sonicated for $15 \mathrm{~min}$ in order to split the agglomerates of polymer spheres. Suspensions prepared in such way have been deposited on Si (100) wafers by dipcoating (DC) or Langmuir-Blodgett (LB) method.

\subsubsection{Dip-coating method}

Aqueous suspensions of PMMA spheres having the following concentrations: 1, 6, 13, and $30 \%$ (weight $\%$ ) were prepared. Si wafers were immersed to the suspension of polymer beads having various concentration using dipcoater with different dipping/emerging speeds: 1, 2, 5 and $10(\mathrm{~mm} / \mathrm{min})$. Afterward, samples were dried in air at room temperature for one day.

\subsubsection{Langmuir-Blodgett method}

For LB applications an aqueous suspensions of poly (methyl methacrylate) spheres were prepared with following concentrations: $0.005,0.1,0.5 \%$ (weight $\%-\mathrm{m} / \mathrm{m}$ ). Before deposition, isotherms presenting the dependence of the surface pressure on the area in the following conditions were recorded:

a. pure deionized water,

b. deionized water with addition of $21 \mu \mathrm{L}$ of $10^{-3} \mathrm{~mol} / \mathrm{L}$ solution of the arachidic acid,

c. suspensions of PMMA beads in deionized water (subphase),

d. suspensions of PMMA beads in deionized water with addition of $21 \mu \mathrm{L}$ of $10^{-3} \mathrm{~mol} / \mathrm{L}$ solution of the arachidic acid. 
Surface pressure versus subphase area graph were traced. Isotherms recorded in these conditions were used to find the value of the target surface pressure. This value was chosen as a target pressure at which PMMA spheres were deposited on $\mathrm{Si}$ wafers. The withdrawal rate of the Si wafer in LB deposition was set up for $5 \mathrm{~mm} / \mathrm{min}$. After deposition, PMMA coatings were dried in air at the room temperature.

\subsection{Synthesis of titania coating}

In order to obtain macroporous titanium dioxide coatings, sol-gel process with titanium (IV) isopropoxide as titania precursor was used. Titanium (IV) isopropoxide $(1.12 \mathrm{~g})$ was added slowly to the isopropyl alcohol $(13.20 \mathrm{~g})$. Subsequently, small amount of $2 \mathrm{M} \mathrm{HCl}(0.04 \mathrm{~g})$ was applied as a catalyst. Resulted mixture was gently stirred for $2 \mathrm{~h}$.

Next, PMMA beads, previously deposited on Si wafers, were infiltrated with titania sol. The immersion rate of the dip-coating process was $5 \mathrm{~mm} / \mathrm{min}$. Every silicon wafer with deposited PMMA beads was kept in the solution of solgel titania precursor for $5 \mathrm{~min}$. Composites obtained in this process were dried in air at room temperature for one day.

\subsubsection{Polymer template removal}

Template removal was conducted in a mixture of acetone and THF (volume ratio 1:1) Deposited titania/PMMA composite coatings were placed into the Soxhlet apparatus equipped with round-bottomed flask $(0.5 \mathrm{~L}$ volume $)$ containing $260 \mathrm{~mL}$ mixture of acetone and THF, reflux condenser and heater. Extraction was carried out by $12 \mathrm{~h}$. After that time titania coatings were recovered and dried at $60{ }^{\circ} \mathrm{C}$ for $20 \mathrm{~min}$.

\subsection{Surface characterization}

PMMA beads and final macroporous coatings were imaged using commercial Atomic Force Microscope-Solver P47 (NT-MDT company) equipped in NSG11 cantilever having force constant $k$ in the range of: $2.5-10 \mathrm{~N} / \mathrm{m}$ and resonance frequency $v=182.1 \mathrm{kHz}$. Measurements were conducted in ambient air at room temperature in the tapping mode. Typical scan areas were $($ in $\mu \mathrm{m} \times \mu \mathrm{m}): 10 \times 10,5 \times 5$, $2 \times 2$ and $1.5 \times 1.5$.

\section{Results and discussion}

\subsection{Characterization of the polymer templates}

The arrangement and size of the prepared PMMA beads play crucial role in further preparation steps of the porous structure. Colloidal crystals built of PMMA beads serve as a template. Therefore thin films built of PMMA beads deposited on $\mathrm{Si}$ wafers have been characterized by Atomic Force Microscope (AFM) in tapping mode.

Our investigations were focused on three aspects. Initially the effect of the synthesis conditions on the size of the PMMA beads was verified. As the novelty in PMMA beads deposition, Langmuir-Blodgett method was applied and compared to dip-coating method. Finally, the effect of the concentration and the substrate immersion/withdrawal velocity in the dip-coating method on the structure and the arrangement of beads was verified.

Since the synthesis was conducted at various concentrations of the initiator and stirring velocities, it was possible to control the size of the polymer beads. In general, the typical size of PMMA beads obtained from synthesis I were: $200-220$ and $120-150 \mathrm{~nm}$ for fraction 1 and 2 respectively. Large size dispersion was achieved for synthesis II in fraction 1, while the size of PMMA beads in fraction 2 in the synthesis II were in the range 320-325 nm (Table 1).

In order to achieve appropriate level of packing of polymer beads on the substrate, the influence of the suspension concentration was investigated in LB and dipcoating methods. Changes in packing density of polymer spheres for various concentrations were observed on AFM images.

In the LB method the concentration of the polymer spheres in the subphase was adjusted for $0.005,0.1$ and 0.5 $\mathrm{wt} \%$. Arachidic acid was used as the auxiliary compound. The concentration of this acid was constant at each case. The subphase is defined as polymer suspension at given concentration placed in the LB trough. It was found, that good packing level of polymer beads was achieved, when the concentration of PMMA spheres in aqueous suspension (in the presence of arachidic acid) was at least $0.1 \mathrm{wt} \%-$ Fig. 1. Fully covered surface was obtained when the concentration of the PMMA beads in the subphase was 0.5 $w t \%$. It is hard to distinguish long-range ordering of the PMMA beads for both concentrations. However, some local arrangement can be distinguished for $0.5 \mathrm{wt} \%$ deposition. It should be highlighted, that the presence of the arachidic acid in the subphase considerably increases the deposition efficiency. If arachidic acid is not added, the

Table 1 Pore size of macroporous titania coatings obtained from templates (polymer spheres) taken from various synthesis and fractions

\begin{tabular}{lll}
\hline $\begin{array}{l}\text { Number of synthesis } \\
\text { and fraction }\end{array}$ & $\begin{array}{l}\text { Diameters of PMMA } \\
\text { beads }(\mathrm{nm})\end{array}$ & $\begin{array}{l}\text { Diameter of } \\
\text { pores }(\mathrm{nm})\end{array}$ \\
\hline Synthesis 1, fraction 1 & $200-220$ & $200-220$ \\
Synthesis 1, fraction 2 & $120-150$ & $130-160$ \\
Synthesis 2, fraction 2 & $320-325$ & $325-330$ \\
\hline
\end{tabular}


surface coverage of the PMMA beads prepared from $0.5 \%$ suspension is very low and corresponds to the surface coverage obtained from $0.005 \%$ in the presence of the acid-Fig. 1. At low concentration only two or three individual beads are observed over the $5 \mu \mathrm{m} \times 5 \mu \mathrm{m}$ surface area. The presence of the arachidic acid molecules enhances the adsorption of polymer beads at the water/air interface by attractive interactions. Consequently, it favors the transfer of polymer beads to the substrate while its withdrawing from the subphase. Figure 2 presents the surface pressure versus area isotherms for various PMMA/ arachidic acid/water systems. It is well visible, that when only PMMA beads are present on the water/air interface, no target pressure can be obtained. These conditions do not enable the transfer of polymer beads onto the substrate. However, if arachidic acid is added, its presence significantly increases the surface pressures up to $40 \mathrm{mN} / \mathrm{m}^{2}$. This value is similar to data obtained by other authors [13]. As a consequence, partially of fully covered by PMMA beads substrate can be obtained respectively for 0.1 and $0.5 \%$ concentrations. Typical isotherm of pure arachidic acid was also recorded and included to Fig. 2.

For DC preparation method, higher concentrations of polymer spheres in deposited aqueous suspensions than in LB method were applied: $1,6,13$, and $30 \%$ respectively. Suspensions were deposited on $\mathrm{Si}$ wafers with constant dipping/withdrawal velocity of $10 \mathrm{~mm} / \mathrm{min}$. Images obtained from the AFM scans clearly demonstrate that the surface density of PMMA beads is higher than in the case of LB method-Fig. 3. Beads are closely packed and for some concentrations $(6 \%)$ are well organized. When $1 \%$ PMMA suspension were deposited on Si wafer islands-like polymer spheres aggregates are visible. Suspensions having concentration of 13 and $30 \%$ resulted in the thick PMMA multilayers exhibited less level of ordering. In further experiments, we also focused on the influence of the dipping/withdrawal rate of the substrate into the suspension, on the packing density choosing $6 \mathrm{wt} \%$ concentration of the PMMA beads.

The effect of the substrate dipping and withdrawal deposition rate on the packing density of PMMA beads was evaluated only in dip-coating method-Fig. 4a.

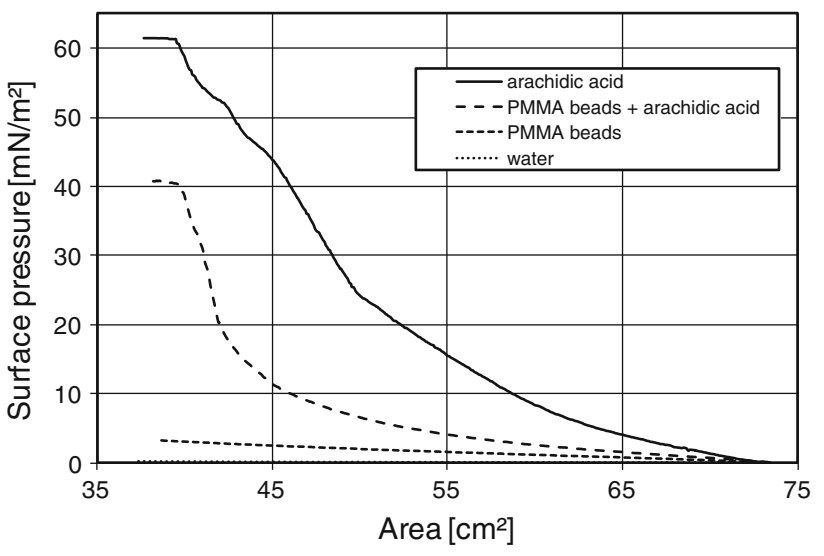

Fig. 2 Surface pressure versus area isotherms for: arachidic acid; PMMA beads and arachidic acid; PMMA beads; pure water

Depositions were performed using $6 \mathrm{wt} \%$ of PMMA beads suspension at following dipping/withdrawal rates: $1,2,5$ and $10(\mathrm{~mm} / \mathrm{min})$. It was found, that the best ordering of the PMMA beads was obtained for $1 \mathrm{~mm} / \mathrm{min}$ Fig. $4 \mathrm{a}$. Detailed analysis of the AFM images of the PMMA layer deposited at $1 \mathrm{~mm} / \mathrm{min}$ revealed local hexagonal arrangement of PMMA spheres-Fig. 4b.

On the basis of obtained results the $6 \mathrm{wt} \%$ concentration of suspension and $1 \mathrm{~mm} / \mathrm{min}$ dipping/withdrawal speed in dip-coating method were chosen for further experiments. PMMA beads deposited at these parameters exhibit good level of arrangement and good surface coverage. They were used as templates allowing to get macroporous titania having well ordered pores.

\subsection{Characterization of the $\mathrm{TiO}_{2}$ macroporous coatings}

Figure 5 presents AFM images of polymer templates deposited using dip-coating method obtained from aqueous suspensions of PMMA beads having various concentrations. This figure presents also macroporous titania after polymer removal. Macroporous titania was obtained after infiltration of polymer template using liquid sol containing titania precursor in dip-coating process. The images of porous titania reveals that their size and density correspond to the size and density of applied beads. It is also visible
Fig. 1 AFM images

$(5 \mu \mathrm{m} \times 5 \mu \mathrm{m})$ of PMMA spheres deposited using different concentrations of the PMMA suspensions in the Langmuir-Blodgett method

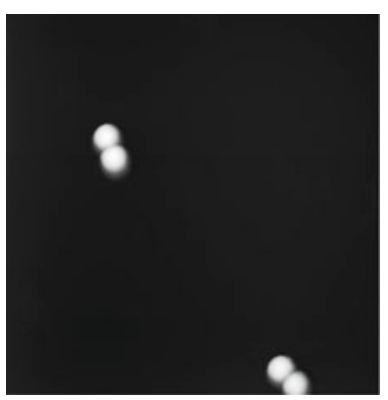

$0.005 \%(\mathrm{~m} / \mathrm{m})$

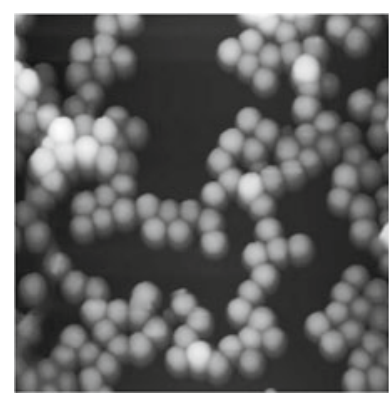

$0.1 \%(\mathrm{~m} / \mathrm{m})$

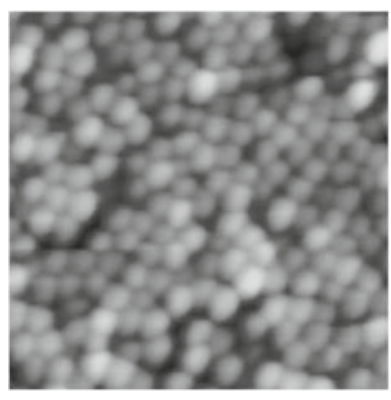

$0.5 \%(\mathrm{~m} / \mathrm{m})$ 

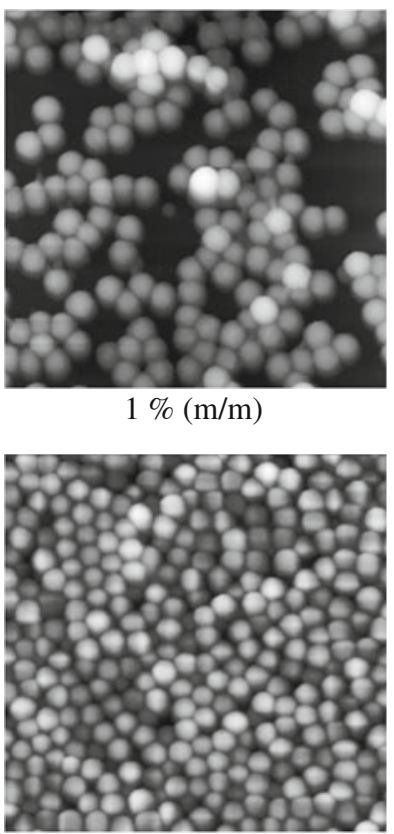

$13 \%(\mathrm{~m} / \mathrm{m})$
$1 \%(\mathrm{~m} / \mathrm{m})$

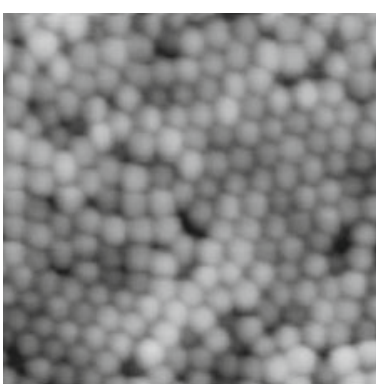

$6 \%(\mathrm{~m} / \mathrm{m})$

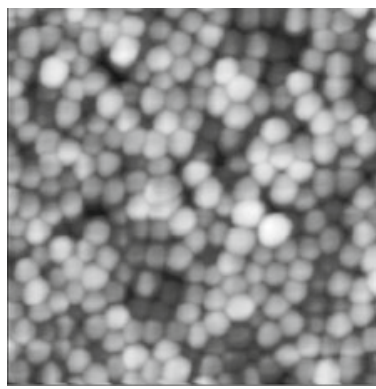

$30 \%(\mathrm{~m} / \mathrm{m})$

Fig. 3 AFM images $(5 \mu \mathrm{m} \times 5 \mu \mathrm{m})$ of PMMA spheres deposited from aqueous suspensions having different concentrations using dipcoating method. Dipping/emerging rate: $10 \mathrm{~mm} / \mathrm{min}$

that even at low PMMA concentrations (1\%), for unevenly distributed beads, porous structures are also created. They look like shells opened from one side loosely embedded side by side. However for higher concentration (30\%) typical macroporous material is created. For some template concentrations $(6 \%)$ hexagonal arrangement of pores is clearly visible.

Figure 6 presents AFM images of macroporous titania coating obtained from polymer template deposited on Si wafer using Langmuir-Blodgett method. The concentration of aqueous PMMA suspension was $0.5 \%$. Closely packed porous structure is visible in the $10 \mu \mathrm{m} \times 10 \mu \mathrm{m}$ area. Distribution of pores in this range is not homogenous. Notwithstanding some small areas of titania coating $(2 \mu \mathrm{m} \times 2 \mu \mathrm{m})$ exhibit hexagonal arrangement of pores-Fig. 6.

Obtained results demonstrate, that both methods of PMMA deposition are effective in preparation of macroporous titania coatings. Distribution of pores in these coatings are not homogenous in every case. However local hexagonal arrangement is possible to achieve for small areas. The mechanism of macroporous titania coatings formation is based on the adsorption of the viscous sol on the PMMA beads. Titania precursor present in the sol is also adsorbed on the polymer beads and consequently undergoes further reactions of hydrolysis and condensation. Since concentration of the titania precursor in the sol is relatively low and the PMMA beads are not closely packed, objects which resemble shells or rings are formed. For higher concentrations of deposited PMMA beads, the volume between individual beads is low. These empty spaces can be easily filled with sol forming stable walls upon polymer removal-Figs. 5, 6 .

Considering the pore size of the obtained macroporous titania coatings, it was found that the diameter of their pores corresponds to the diameter of PMMA beads used as
Fig. 4 AFM images

$(5 \mu \mathrm{m} \times 5 \mu \mathrm{m})$ of PMMA spheres deposited from $6 \%(\mathrm{~m} /$ $\mathrm{m})$ aqueous suspension, with different dipping/emerging rates and the dip-coating method (a). AFM image $(1.5 \mu \mathrm{m} \times 1.5 \mu \mathrm{m})$ of the polymer template with visible hexagonal ordered spheres (b)

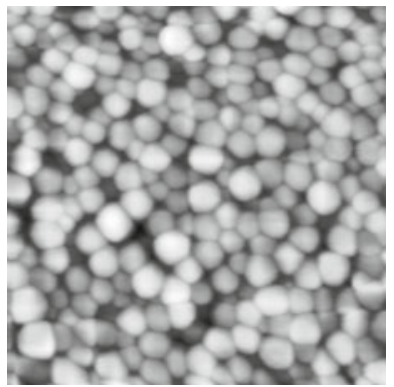

$10 \mathrm{~mm} / \mathrm{min}$.

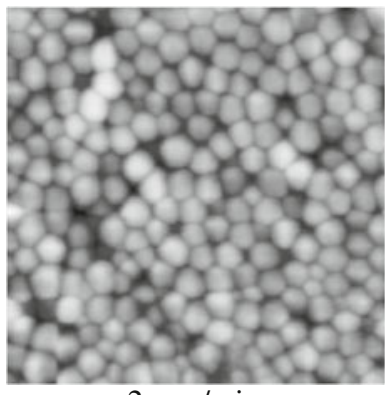

$2 \mathrm{~mm} / \mathrm{min}$

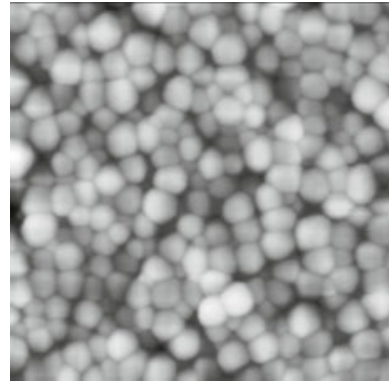

$5 \mathrm{~mm} / \mathrm{min}$

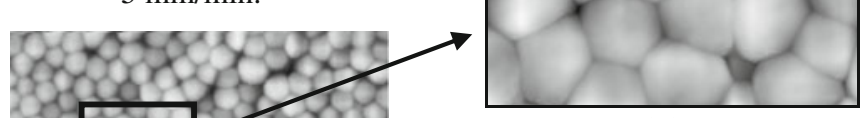

(b)

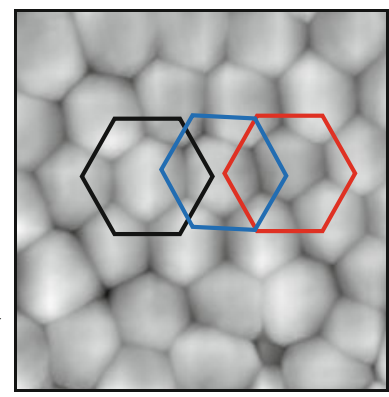

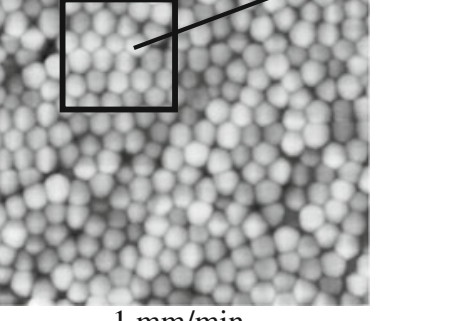

$1 \mathrm{~mm} / \mathrm{min}$.

(a) 
Fig. 5 AFM images of polymer templates (prepared by different concentrations of aqueous suspensions using dip-coating method) and obtained macroporous titania.

$(5 \mu \mathrm{m} \times 5 \mu \mathrm{m})$ images of polymer templates (a);

$(5 \mu \mathrm{m} \times 5 \mu \mathrm{m})$ images (b) and $(2 \mu \mathrm{m} \times 2 \mu \mathrm{m})$ images (c) of macroporous titania obtained from above polymer

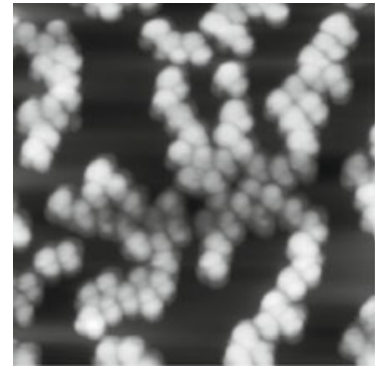

$1 \%$

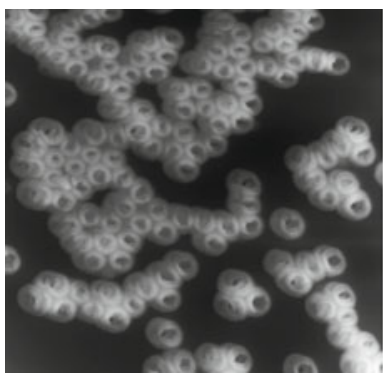

$1 \%$

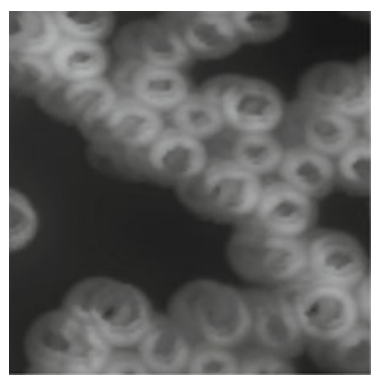

(a)

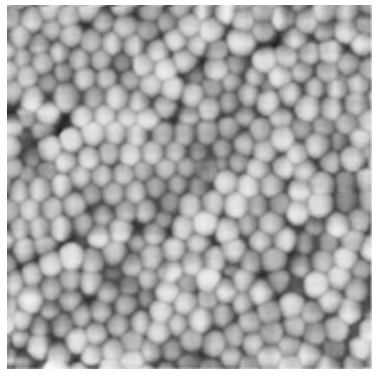

$6 \%$

(b)

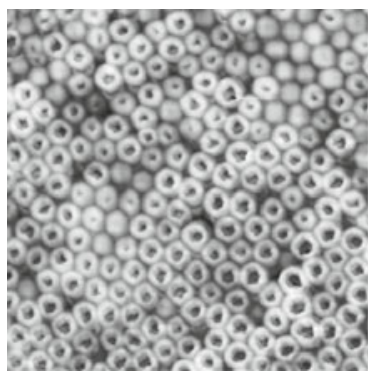

$6 \%$

(c)

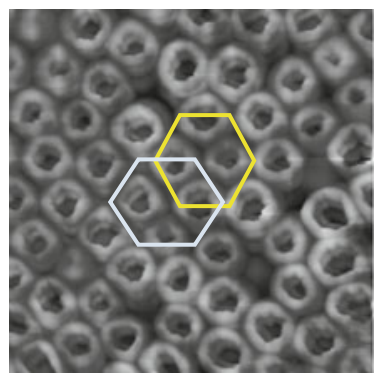

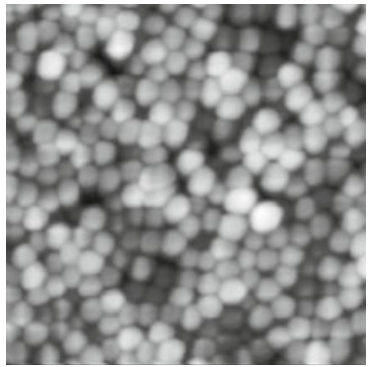

$30 \%$

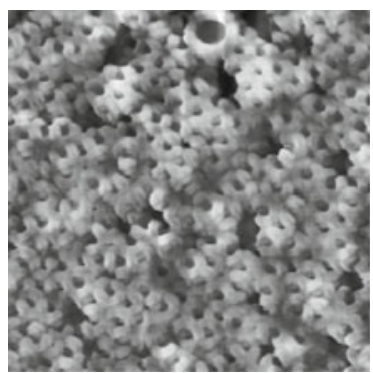

$30 \%$

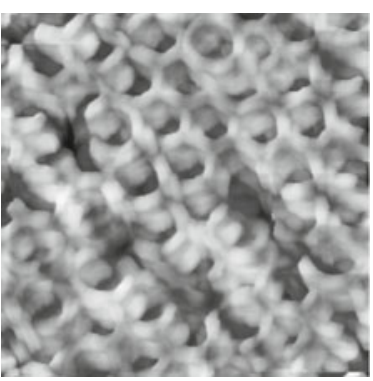

Fig. 6 AFM images

$(10 \mu \mathrm{m} \times 10 \mu \mathrm{m}$ and

$2 \mu \mathrm{m} \times 2 \mu \mathrm{m}$ ) of macroporous titania obtained from polymer template (prepared by $0.5 \%$ concentration of aqueous suspension using LangmuirBlodgett method) exhibiting local hexagonal order of pores
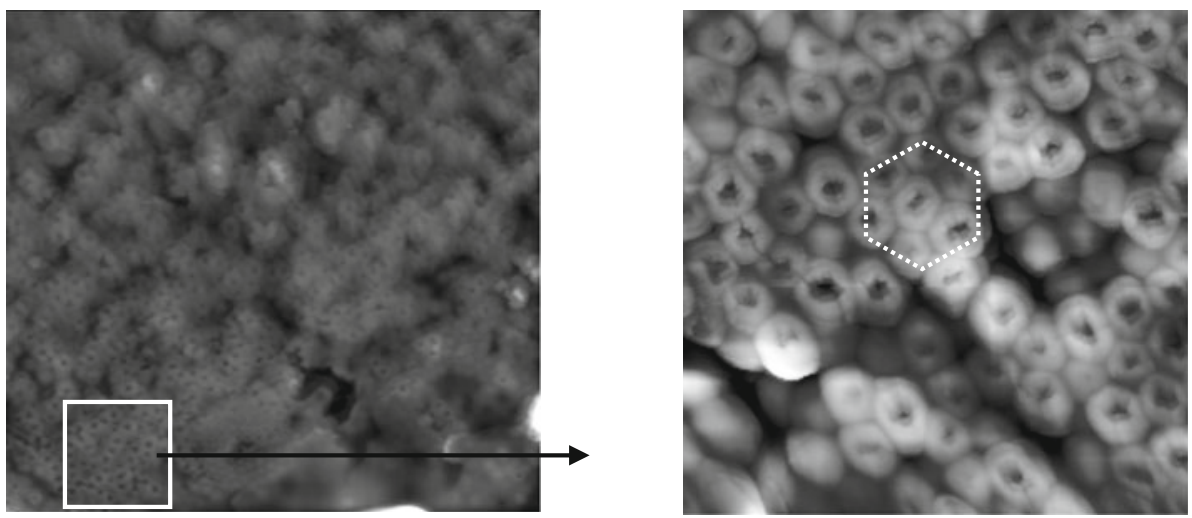

a template. In general, the pore size of macroporous titania is in the range of $130-330 \mathrm{~nm}$. Figure 7 presents AFM images of macroporous titania and in plane cross-sections showing diameters of pores obtained using dip-coating (Fig. 7a, b) and Langmuir-Blodgett methods (Fig. 7c). These images reveal very good correlation between the pore size of macroporous materials and PMMA beads used as the template. Detailed distribution of pores size and corresponding diameters of PMMA beads achieved from various fractions are collected in Table 1. Data obtained in our experiments demonstrate that the pore size can be easily adjust by the appropriate selection of the polymer 

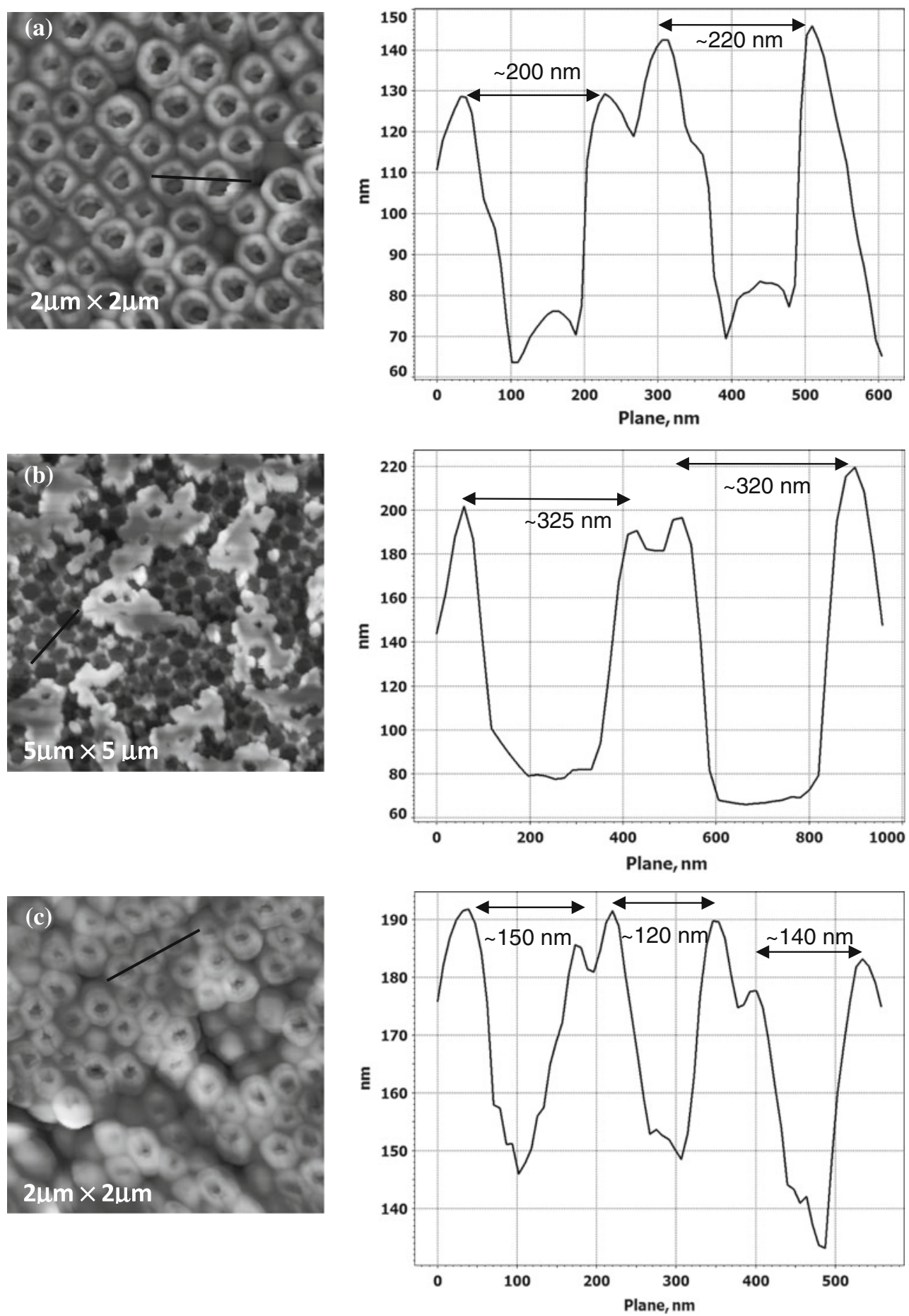

Fig. 7 AFM images $(5 \mu \mathrm{m} \times 5 \mu \mathrm{m}$ and $2 \mu \mathrm{m} \times 2 \mu \mathrm{m})$ of macroporous titania (with plane cross—diameters of their pores) obtained using: dipcoating (a, b) and Langmuir-Blodgett method (c)

template. This issue is important especially form the point of view of application of such materials in optoelectronic devices e.g. photonic crystals.

AFM technique enables only imaging the top surface layer of the scanned material. Therefore additional simple experiment allowing the view inside the porous structure was conducted. This experiment consisted on the gently sticking of the adhesive tape to the surface of macroporous titania coatings after extraction and removing this tape with attached part of the coating. Most of the AFM users are familiarized to this procedure. It is often used in cleaning of the muscovite surface substrates for a various AFM 
experiments. Resulted "fresh" surface was consequently scanned-Fig. 7b. This experiment revealed partially uncovered porous structure with widely open pores having the diameter around $320 \mathrm{~nm}$. Part of pores is not visible. They are clogged by the squeezed material of the coating. This experiment demonstrate also that obtained porous titania coatings exhibit multilayer structure. In such type of materials, important porosity is visible not only on the surface, but also inside the coating.

In our opinion, experiments presented in this work can be continued in several directions, which cover such areas like application of processes in supercritical conditions, binary template systems and plasma etching methods.

Recently highly ordered macroporous titania was produced in supercritical $\mathrm{CO}_{2}$ using the sol-gel process [15]. Regularly packed polystyrene (PS) particles having the diameter of $715 \mathrm{~nm}$ were used as a template. The PS template was subsequently impregnated with the mixture of supercritical $\mathrm{CO}_{2}$, titanium tetraisopropoxide, ethanol and distilled water. The advantage of preparation of macroporous titania in supercritical conditions is high-speed, high-quality inverse opal formation, complete infiltration of pores with desired compound and preventing of shrinkage-induced cracking. Inverse opals inorganic oxide materials, including titania, having highly ordered porous structure, can be applied in tissue engineering, catalysis, photonic crystal devices and energy storage devices due to its photonic band gap.

Preparation of the $\mathrm{TiO}_{2}$ inverse opals can be also conducted using binary template system [16]. Polystyrene binary colloidal crystals having the diameter of 170 and $1,000 \mathrm{~nm}$ and moisture stable $\mathrm{TiO}_{2}$ precursor were used. Additionally, application of sandwich vacuum method of the precursor infiltration provided $\mathrm{TiO}_{2}$ binary inverse opals without overlayers in large domains, exhibiting enhanced photocatalytic activity as compared to $\mathrm{TiO}_{2}$ nanoparticles.

Also recently gradient colloidal photonic crystals (GCPCs) were prepared by plasma etching method [17]. The gradient (along the surface normal) can be controlled by appropriate plasma treatment. Application of plasma provides larger voids for filling with other materials. Such approach enables further tuning of the stop band of the GCPCs and makes them more suitable to construct tunable photonic crystals compared with CPCs.

\section{Conclusions}

This paper presents two alternative preparation methods of macroporous titania coatings. Both methods are based on the application of polymer PMMA beads, which serve as template for synthesis of macroporous titania coatings.
PMMA beads were obtained by emulsifier-free polymerization. In the first method, as the novelty, the LangmuirBlodgett technique was applied in deposition of polymer beads on the solid substrate. For comparative studies of PMMA deposition, dip-coating method was used. Infiltration of resulted PMMA films with titania precursor was the same in both cases.

It was found that Langmuir-Blodgett technique can be effective in the formation of polymer templates only when arachidic acid is present in the subphase. If it is not, the application possibilities of this technique are strongly limited. For both methods: Langmuir-Blodgett and dip-coating the effect of the concentration on the final arrangement of polymer beads is visible. However, in the case of the LB method the concentration of polymer beads in the suspension is much lower than in the dip-coating method. Additionally, in the dip-coating method, slow immersion and withdrawal rates enable preparation of more ordered structures. We found good correlation between the size and the pore arrangement of the final materials and the size and arrangement of polymer templates.

This work can be the starting point for further experiments leading to better arrangement of pores and applied studies of resulting macroporous materials. Macroporous periodic structures may have wide applications in solar cells, photochemistry, photocatalysis, tissue engineering, photonic crystal devices (color displays, photonic sensors) and energy storage devices due to its photonic band gap.

Acknowledgments This work was supported by the Ministry of Science and Higher Education of Poland. Grant number: N N507 497538 .

Open Access This article is distributed under the terms of the Creative Commons Attribution License which permits any use, distribution, and reproduction in any medium, provided the original author(s) and the source are credited.

\section{References}

1. L. Zhi-cheng, R. Wei-dong, J. Nan, R. Lu-quad, C. Qian, Z. Bing, J. Bionic Eng. 3, 059 (2006)

2. S. Reculusa, P. Massé, S. Ravaine, J. Colloid Interf. Sci. 279, 471 (2004)

3. M.C. Carbajo, E. Enciso, M.J. Torralvo, Colloid Surface A 293, 72 (2007)

4. Y. Sakka, F. Tang, H. Fudouzi, T. Uchikoshi, Sci. Technol. Adv. Mat. 6, 915 (2005)

5. F. Tang, T. Uchikoshi, Y. Sakka, Mater. Res. Bull. 41, 268 (2006)

6. M.A. Al-Daous, A. Stein, Chem. Mater. 15, 2638 (2003)

7. X. Wang, P. Dong, S. Chen, Acta Phys.-Chim. Sin. 22, 831 (2006)

8. B.T. Holland, C.F. Blanford, T. Do, A. Stein, Chem. Mater. 11, 795 (1999)

9. B.T. Holland, C.F. Blanford, A. Stein, Science 281, 538 (1998) 
10. H. Yan, K. Zhang, C.F. Blanford, L.F. Francis, A. Stein, Chem. Mater. 13, 1374 (2001)

11. B. Gates, Y. Yin, Y. Xia, Chem. Mater. 11, 2827 (1999)

12. H. Yan, C.F. Blanford, B.T. Holland, M. Parent, W.H. Smyrl, A. Stein, Adv. Mater. 11, 1003 (1999)

13. İ. Çapan, T. Tanrisever, Optoelectron. Adv. Mater. 4, 1278 (2010)

14. D. Zou, S. Ma, R. Guan, M. Park, L. Sun, J.J. Aklonis, R. Salovey, J. Polym. Sci. A1(30), 137 (1992)
15. H.M. Yu, J.-H. Yim, K.Y. Choi, J.S. Lim, J. Supercrit. Fluid 67, $71(2012)$

16. Z. Cai, J. Teng, Z. Xiong, Y. Li, Q. Li, X. Lu, X.S. Zhao, Langmuir 27, 5157 (2011)

17. H. Huang, J. Chen, Y. Yua, Z. Shia, H. Möhwald, G. Zhang, Colloid Surface A 428, 9 (2013) 